Original Article (short paper)

\title{
ADRB2 GIn27Glu polymorphism influenced changes in leptin but not body composition or metabolic and other inflammatory parameters after twelve weeks of combined training in overweight adolescents
}

\author{
Neiva Leite ${ }^{1}$, Larissa Rosa da Silva ${ }^{1}$, Íncare Correa de Jesus ${ }^{1}$, Wendell Arthur Lopes ${ }^{2}$, Claudia Regina Cavaglieri ${ }^{3}$, \\ Cássio Leandro Consentino ${ }^{4}$, Rosana Bento Radominski ${ }^{4}$, Ricardo Lehtonen Rodrigues de Souza ${ }^{4}$, Luciane Viater \\ Tureck ${ }^{5}$, Lupe Furtado-Alle \\ ${ }^{1}$ Universidade Federal do Paraná, UFPR, Curitiba, PR, Brazil; ${ }^{2}$ Universidade Estadual de Maringá, UEM, \\ Maringá, PR, Brazil; ${ }^{3}$ Universidade Estadual de CAmpinas, UNICAMP, Campinas, SP, Brazil; ${ }^{4}$ Universidade \\ Federal do Paraná, UFPR, Curitiba, PR, Brazil; ${ }^{5}$ Universidade Tecnológica Federal do Paraná, UTFPR, \\ Ponta Grossa, PR, Brazil; ' ${ }^{6}$ Universidade Federal do Paraná, UFPR, Curitiba, PR, Brazil.
}

\begin{abstract}
Aim: To compare the anthropometric, metabolic, and inflammatory parameters of overweight adolescents after 12-weeks of resistance and aerobic training (CT), taking into account the Gln27Glu polymorphism of the $\beta 2$ adrenergic receptor $(A D R B 2)$ gene. Methods: Forty-seven adolescents $(15.05 \pm 1.07 \mathrm{y})$ were assigned to one of four groups, according to the presence or absence of the Glu27 allele: CT (CarrierT n=11; NoncarrierT $n=11$ ) or control (CarrierC $\mathrm{n}=13$; NoncarrierC $\mathrm{n}=12$ ). Body composition, abdominal fat, maturation, fitness, metabolic and lipid profile, inflammatory markers were assessed. The CT consisted of six resistance exercises, followed by $30 \mathrm{~min}$ of walking/ running at $50-80 \% \mathrm{VO}_{2 \max }$, totaling $60 \mathrm{~min} / \mathrm{session}$, three times a week. A mixed-model factorial ANOVA was used to compare variables at baseline and after 12-weeks. Results: TC was effective in reducing total fat mass (NoncarrierT $\mathrm{ES}=.45$, CarrierT $\mathrm{ES}=.27$ ) and subcutaneous abdominal fat (NoncarrierT $\mathrm{ES}=.48$, CarrierT $\mathrm{ES}=.46$ ) and increasing lean mass (NoncarrierT ES=.58, CarrierT ES=.60) and fitness. CarrierT group showed a reduction in leptin (ES=.49). Conclusion: The responses of body composition and physical fitness to TC were not influenced by the presence of the Gln27Glu polymorphism. However, only the Glu27 allele carriers showed reductions in leptin after 12-weeks. Besides, a lack of intervention caused obesogenic effects, especially in Glu27carriers.
\end{abstract}

Keywords: genetics, exercise, obesity, adolescents, metabolism.

\section{Introduction}

Obesity is considered a chronic and progressive disease that is associated with metabolic and cardiovascular complications $\mathbf{s}^{1,2}$. Its treatment requires a combination of regular physical activity and healthy eating habits, in order to achieve weight reduction and long-term weight loss maintenance ${ }^{3,4}$. The challenge is to monitor the therapeutic response of obese individuals, who are under the influence of environmental, hormonal and genetic factors that interfere in body weight reduction and control ${ }^{5}$.

Genetic factors, such as the presence of polymorphisms in adrenergic receptor (ADR) encoding genes, have been associated with changes in energy regulation and expenditure ${ }^{6}$. In fact, polymorphisms in the gene encoding $\beta 2$ adrenergic receptor (ADRB2) may change the thermogenic effects of catecholamines, being associated with the presence of obesity and weight regain ${ }^{7,8}$, and may also alter lipolysis ${ }^{9}$. Therefore, allelic variants in genes associated with energy expenditure may negatively influence the reduction of adipose tissue ${ }^{10}$ and weight reduction ${ }^{11}$.

Intervention programs that include physical exercise and restrictive diet have effective results in weight loss in obese adults $^{12}$. However, in adolescents, physical activities associated with nutritional guidance for healthy and balanced eating habits is recommended ${ }^{13}$, which can promote the reduction of body fat ${ }^{14}$, abdominal obesity and insulin resistance ${ }^{15,16}$, as well as the reduction of obesity-associated chronic inflammation ${ }^{14}$.

Specific genetic variants may interfere in the metabolic response to training, contributing to the variability in individual response to exercise training ${ }^{17}$. In this context, the polymorphism Gln27Glu (rs1042714) in the ADBR2 gene, in which substitution of a cytosine for a guanine leads to amino acid changes at protein position 27 (substitution of glutamine (Gln) for glutamic acid (Glu), appears to be important. In adults, although exercise increases the activity of the sympathetic nervous system, this response was impaired in obese women carriers of the $27 \mathrm{Glu}$ allele ${ }^{18}$, whereas obese subjects with homozygous genotype (Gln27Gln) benefitted from physical activity in order to reduce weight ${ }^{19}$.

In the treatment of obese adolescents, combined exercises has been used with excellent metabolic ${ }^{20}$ and inflammatory responses ${ }^{14}$ and seems to more effective in improving the visceral fat than aerobic training alone ${ }^{21}$, and induce the same effects in fitness that isolated aerobic and resistance training $\mathrm{do}^{22}$. Nevertheless, the impact that the Glu27 allele may have on inflammatory parameters in response to combined training have not been assessed. Thus, this study aimed to compare anthropometric, metabolic and inflammatory parameters of overweight adolescents, carriers and noncarriers of the Glu27 allele of $A D R B 2$ gene, after 12 weeks of combined training. 


\section{Methods}

The study was composed of 47 overweight adolescents, aged 12 to 16 years. The sample size was calculated by the $\mathrm{G}^{*}$ Power3 software considering an $\alpha$ of $0.05, \beta$ of 0.8 and minimal effect size of 0.2 , according to the changes in the $\mathrm{VO}_{2 \max }$ of overweight girls who underwent combined training for 12 weeks ${ }^{14}$. The minimum sample size was of six participants per each groups (total $n=24$ ).

They were genotyped for Gln27Glu (rs1042714, C>G) polymorphism of the $A D B R 2$ gene. The recessive and additive allelic interaction models were also tested, but the dominant model was consistent with our results. Thus, carriers of Gln27Glu and Glu27Glu genotypes were grouped (Glu27 carriers), whereas those with the Gln27Gln genotype were considered noncarriers.

After the genotypes had been identified, the subjects were grouped according to the presence or absence of the Glu27 allele, and then randomly assigned to the training or the control group. Thus, the subjects were divided into four groups: a) CarrierT-Training group consisting of ADRB2 Glu27 allele carriers $(n=11)$; b) CarrierC - Control group consisting of ADRB2 Glu27 allele carriers $(\mathrm{n}=13)$; c) NoncarrierT - Training group consisting of Glu27 allele noncarriers ( $\mathrm{n}=11)$; and d) NoncarrierC - Control group consisting of Glu27 allele noncarriers $(n=12)$. The NonCarrierC and CarrierC groups were used for the clinical monitoring of the physiological changes that might occur within the 12 weeks, independently of intervention.

The inclusion criteria were BMI Z-score $\geq 1$; Tanner stage 4 or 5; being at a stable weight for two months or longer; not being using anorectic drugs or other drugs that may interfere on weight control and hyperinsulinemia; and having had no exercise in the last 6 months. Exclusion criteria were medical contraindication to exercise; changes in TSH levels; and having participated in less than $70 \%$ of the training sessions.

All subjects were informed of the objectives, experimental procedures and possible protocol events. All participants signed an assent form, while their parents or legal guardians signed a consent form previously approved by the Ethics Committee of the Federal University of Paraná (protocol number 2460.067/2011-03).

\section{Assessments}

All participants were assessed twice by a multidisciplinary team, at the baseline and after 12 weeks of treatment.

\section{Anthropometry}

Height was measured to the nearest $0.1 \mathrm{~cm}$, using a wall stadiometer, and weight was measured in kilograms $(\mathrm{kg})$ on a platform scale of the brand Filizola, with $150 \mathrm{~kg}$ capacity and accurate to $100 \mathrm{~g}$. Waist circumference was measured to the nearest 0.1 $\mathrm{cm}$, using a flexible non-elastic tape parallel to the ground and placed midway between the iliac crest and the last rib $^{23}$.

Body Mass Index was calculated by body mass (kg) divided by height squared $\left(\mathrm{m}^{2}\right)$. The z-score was calculated using the World Health Organization AnthroPlus software. Adolescents with a BMI z-score $\geq 1$ were considered overweight.

\section{Body Composition}

Body composition was assessed by dual-energy X-ray absorptiometry (DXA), using a Lunar ${ }^{\mathrm{TM}}$ Prodigy, according to the protocol previously described ${ }^{24}$. The analysis and quantification of bone mineral content and underlying tissues, total fat mass and fat free mass were performed using the enCore 2008 software, version 12.30 . The assessment was performed by a single trained examiner and the intra-observer coefficient of variation was $0.1 \%$ for total mass; $2.4 \%$ for fat mass; $3.06 \%$ for trunk fat mass; and $1.64 \%$ for the fat percentage.

\section{Muscular Strength}

Muscular strength was assessed by 1RM testing in the bench press, leg press and arm curl, after a period of familiarization with the resistance-training exercises. The 1RM protocol was conducted according to Brown and Weir ${ }^{25}$. The tests were later used for the prescription of the intensity of the exercises performed during the intervention with resistance training.

\section{Cardiorespiratory Fitness}

Cardiorespiratory fitness was assessed with a treadmill test, using the X-Fit 7 Power Treadmill and a breath-by-breath gas analyzer $\left(\mathrm{K} 4 \mathrm{~b}^{2}\right.$, Cosmed, Italy). Prior to the beginning of the test, in order to warm up and get used to the treadmill, the subjects walked on it for 2 minutes. We used the modified treadmill ramp protocol, starting at $4 \mathrm{~km} / \mathrm{h}$ and $1 \%$ slope, with increments of $0.3 \mathrm{~km} / \mathrm{h}$ every 30 seconds and a constant slope of $1 \%$ until maximum effort was achieved ${ }^{26}$. The test was considered maximum if at least two of the following criteria were met: a) exhaustion or inability to maintain the required speed; b) HR at or above $200 \mathrm{bpm}$, and c) respiratory exchange ratio (RER) equal to or greater than one. Maximum oxygen consumption $\left(\mathrm{VO}_{2 \max }\right.$ was calculated as described above ${ }^{22}$.

\section{Biochemical analyses}

Blood samples were collected by a qualified professional in the morning, after 12 hours of fasting. Post-training blood collection occurred 72 hours after the last training session. For the cytokine assays, the serum was extracted and frozen at $-80^{\circ} \mathrm{C}$ for later analysis. The interleukin-6 (IL-6), C-reactive protein (CRP), leptin and adiponectin serum levels were determined by ELISA (solid-phase, enzyme-linked immunosorbent assay), using high sensitivity kits (R \& D Systems, Minneapolis, USA) and following the manufacturer's instructions.

Total cholesterol (TC), High Density Lipoprotein (HDL-c) and triacylglycerol (TAG) levels were determined by the enzymatic colorimetric test and Low Density Lipoprotein (LDL-c) levels were estimated using the equation by Friedewald ${ }^{27}$. Blood glucose was determined by an enzymatic method, while insulin was measured by chemiluminescent immunometric immunoassay in an automated analyzer. Insulin resistance (IR) and insulin 
sensitivity (IS) were assessed using the Homeostasis Model Assessment (HOMA) ${ }^{28}$ and the Quantitative Insulin Sensitivity Check Index (QUICKI), respectively ${ }^{29}$.

\section{Genetic analyses}

Leukocyte DNA extractions were performed using the method by Lahiri and Nurnberger ${ }^{30}$. The obtained DNA was diluted to a final concentration of $20 \mathrm{ng} / \mathrm{uL}$. ADBR2 Gln27Glu polymorphism was genotyped using a TaqMan allelic discrimination assay and performed on a 7500TM Real-Time PCR System (Applied Biosystems, Foster City, CA, USA). The following PCR protocol was used: step 1,2 minutes at $50^{\circ} \mathrm{C}$; step 2,10 minutes at $95^{\circ} \mathrm{C}$; and step 3,50 cycles of 15 seconds at $95^{\circ} \mathrm{C}$ followed by 1 minute at $62^{\circ} \mathrm{C}$.

\section{Subcutaneous and visceral abdominal ultrasonography}

Visceral and subcutaneous adiposity measurements were performed according to Vlachos, Hatziioannou, Perelas, Perrea ${ }^{31}$, using a portable ultrasound device of the brand GE, model Logiq Book XP, for high-resolution ultrasonography, with $8 \mathrm{MHz}$ linear transduction. The intra-observer coefficients for visceral and subcutaneous fat measures were $1.92 \%$ and $.36 \%$, respectively.

\section{Dietary assessment}

Nutritional assessment was evaluated by a $24-\mathrm{h}$ food record $(\mathrm{R} 24 \mathrm{~h})^{32}$, before and after the intervention. The instrument was applied by a nutritionist, in two non-consecutive days. The recalls were tabulated on the Diet Pro® 5.5 software to obtain the information related to total energy consumption (TEC), carbohydrates, proteins and lipids, and were presented in kilocalories and grams. The participants were requested not to change their usual food intake during the intervention.

\section{Intervention Program}

The combined training (CT) protocol consisted of resistance training (RT) and aerobic training (AT) performed in the same session, three times a week and divided into three phases (P1, P2 and P3), each consisting of 4 weeks of training. RT was composed of six exercises and aerobic training AT consisted of walking/running in an athletic track (Table 1). The characterization of the training sessions and intensity progression has been previously described ${ }^{14}$. The RT workloads were adjusted weekly. The participants were encouraged to perform the greatest number of repetitions when they came to the last set of each exercise, maintaining the same range of motion and execution speed previously determined. The workloads were increased by $1 \mathrm{~kg}$ for lower body and $1 / 2 \mathrm{~kg}$ for upper body for each repetition performed over the established training protocol in the last set of the last training session of the week.

\section{Statistical analysis}

Normal distribution of data was verified by Shapiro-Wilk test. The chi-square test was used to compare proportions between genders by group. Due to the small number of subjects per group, a natural logarithm was applied to normalize the data. Between-group comparisons were conducted by using ANOVA one-way for parametric data and the Kruskal-wallis test for nonparametric data at baseline. Statistical significance for multiple comparisons was defined at the corrected $\alpha=.0083$ for baseline comparisons. Comparisons of anthropometric, fitness, body composition, ultrasound and metabolic parameters between baseline and post-treatment (time factor) and between groups (group factor) were performed using a mixed-model factorial ANOVA. The equality of variance was assessed by the Levene's test. Observed effect size (ES) for each group was calculated by Cohen's $d^{33}$. The analysis of the changes between the NoncarrierC and CarrierC groups was performed based on the observed magnitude of the effect ${ }^{33}$. Comparisons between subjects were performed by Wilcoxon signed-ranks test with

Table 1. Combined training programme.

\begin{tabular}{|c|c|c|c|c|c|c|c|c|c|c|c|c|c|c|c|c|c|}
\hline \multirow{2}{*}{$\begin{array}{c}\text { Resistance } \\
\text { Exercise }\end{array}$} & \multicolumn{5}{|c|}{ P1 (1-4 wk) } & \multicolumn{6}{|c|}{ P2 (5-8 wk) } & \multicolumn{6}{|c|}{ P3 (9-12 wk) } \\
\hline & $\begin{array}{l}\text { Set } \\
\text { (n) }\end{array}$ & $\begin{array}{c}\text { Rep } \\
\text { (n) }\end{array}$ & $\begin{array}{l}\text { Load } \\
(\mathrm{RM})\end{array}$ & \multicolumn{2}{|c|}{$\begin{array}{c}\text { Rest } \\
(\text { min) }\end{array}$} & $\begin{array}{l}\text { Set } \\
\text { (n) }\end{array}$ & & $\begin{array}{l}\text { Rep } \\
\text { (n) }\end{array}$ & \multicolumn{2}{|c|}{$\begin{array}{l}\text { Load } \\
\text { (RM) }\end{array}$} & $\begin{array}{l}\text { Rest } \\
\text { (min) }\end{array}$ & $\begin{array}{l}\text { Set } \\
\text { (n) }\end{array}$ & & $\begin{array}{c}\text { Rep } \\
\text { (n) }\end{array}$ & \multicolumn{2}{|c|}{$\begin{array}{l}\text { Load } \\
\text { (RM) }\end{array}$} & $\begin{array}{l}\text { Rest } \\
\text { (min) }\end{array}$ \\
\hline Leg press & 3 & 10 & 10RM & \multicolumn{2}{|c|}{1} & 3 & & 8 & \multicolumn{2}{|c|}{$8 \mathrm{RM}$} & 1.5 & 3 & & 6 & \multicolumn{2}{|c|}{$6 \mathrm{RM}$} & 2 \\
\hline Leg extension & 3 & 10 & $10 \mathrm{RM}$ & \multicolumn{2}{|c|}{1} & 3 & & 8 & \multicolumn{2}{|c|}{$8 \mathrm{RM}$} & 1.5 & 3 & & 6 & \multicolumn{2}{|c|}{$6 \mathrm{RM}$} & 2 \\
\hline Leg curl & 3 & 10 & $10 \mathrm{RM}$ & \multicolumn{2}{|c|}{1} & 3 & & 8 & \multicolumn{2}{|c|}{$8 \mathrm{RM}$} & 1.5 & 3 & & 6 & \multicolumn{2}{|c|}{$6 \mathrm{RM}$} & 2 \\
\hline Bench press & 3 & 10 & $10 \mathrm{RM}$ & \multicolumn{2}{|c|}{1} & 3 & & 8 & \multicolumn{2}{|c|}{$8 \mathrm{RM}$} & 1.5 & 3 & & 6 & \multicolumn{2}{|c|}{$6 \mathrm{RM}$} & 2 \\
\hline Lateral pull-down & 3 & 10 & $10 \mathrm{RM}$ & \multicolumn{2}{|c|}{1} & 3 & & 8 & \multicolumn{2}{|c|}{$8 \mathrm{RM}$} & 1.5 & 3 & & 6 & \multicolumn{2}{|c|}{$6 \mathrm{RM}$} & 2 \\
\hline Arm Curl & 3 & 10 & $10 \mathrm{RM}$ & \multicolumn{2}{|c|}{1} & 3 & & 8 & \multicolumn{2}{|c|}{$8 \mathrm{RM}$} & 1.5 & 3 & & 6 & \multicolumn{2}{|c|}{$6 \mathrm{RM}$} & 2 \\
\hline $\begin{array}{l}\text { Aerobic } \\
\text { Exercise }\end{array}$ & $\begin{array}{l}<\mathrm{VT} \\
(\min )\end{array}$ & $\begin{array}{l}=\mathrm{VT} \\
(\mathrm{min})\end{array}$ & $\begin{array}{c}\mathrm{VT}-\mathrm{RCP} \\
\quad(\min )\end{array}$ & $\begin{array}{c}=\mathrm{RCP} \\
(\mathrm{min})\end{array}$ & $\begin{array}{l}<\mathrm{VT} \\
\text { (min) }\end{array}$ & $\begin{array}{l}<\mathrm{VT} \\
(\min )\end{array}$ & $\begin{array}{l}=\mathrm{VT} \\
(\min )\end{array}$ & $\begin{array}{r}\mathrm{VT} \\
\quad(\mathrm{n}\end{array}$ & & $\begin{array}{c}=\mathrm{RCP} \\
(\mathrm{min})\end{array}$ & $\begin{array}{l}<\mathrm{VT} \\
(\text { min) }\end{array}$ & $\begin{array}{l}<\mathrm{VT} \\
\text { (min) }\end{array}$ & $\begin{array}{l}=\mathrm{VT} \\
(\min )\end{array}$ & $\begin{array}{ll} & \mathrm{VT} \\
& (\mathrm{n}\end{array}$ & $\begin{array}{l}\mathrm{RCP} \\
\text { n) }\end{array}$ & $\begin{array}{c}=\mathrm{RCP} \\
(\mathrm{min})\end{array}$ & $\begin{array}{l}P \quad<\mathrm{VT} \\
(\min )\end{array}$ \\
\hline $\begin{array}{l}\text { Walking/ } \\
\text { Running }\end{array}$ & 5 & 10 & 10 & & 5 & 5 & - & & & 10 & 5 & 3 & - & & & 10 & 5 \\
\hline
\end{tabular}

$\mathrm{P}=$ phasesVT - Ventilatory threshold; RCP - Respiratory Compensation point; $\mathrm{RM}$ - maximal repetition. 
split file by group for variables that did not show normality. The $\mathrm{p} \leq .05$ value was considered statistically significant.

\section{Results}

All adolescents were in the post-pubertal stage. There were no differences between groups on anthropometric, body composition, metabolic, and physical fitness variables at baseline. The NonCarrierT group had higher levels of adiponectin than the NoncarrierC group, but lower levels than the CarrierC group, while the CarrierT group had higher levels of adiponectin than the NoncarrierC group and the NoncarrierT group. IL-6 levels were greater in the NoncarrierC group than in the CarrierT group, but smaller when compared to the NoncarrierT group. As for the IL-10 levels, the NoncarrierT group had higher levels when compared to the other three groups. Moreover, the NoncarrierC group had higher levels than the CarrierT group (Table 2).

After 12 weeks of training, there were no differences between
NoncarrierT and CarrierT with regards to BM, height, BMI, WC, SFAT, GLUC, TC, HDL-c, LDL-c, VLDL, TG, Leg press and arm curl. The NoncarrierT group had a higher frequency of subjects with increased INS levels $(Z=-2.401 ; p=.016)$ and reduced QUICKI values $(Z=-2.045 ; p=.041)$ than the CarrierT group (Table 3$)$.

Both groups showed a reduction in FM and TFAT. Nevertheless, the CarrierT group showed a possibly beneficial effect, whereas for the NoncarrierT group the effect was probably trivial. VFAT reduction and LM increase were similar between groups, since both showed a probably trivial effect. Both groups showed possibly beneficial effects for increased bench press strength and reduced subcutaneous abdominal fat. However, the CarrierT group showed a possibly beneficial effect for $\mathrm{VO}_{2 \max }$, whereas the NoncarrierT only exhibited a probably trivial effect (Figure 1).

On the inflammatory markers, the CarrierT group showed an increase in IL-6 levels with an unclear effect size, whereas the NonCarrierT group had a reduction in IL-6 levels after 12 weeks of training, with a possibly beneficial effect. Of the

Table 2. Baseline characteristics of the training group and control group, both divided into carrier and noncarrier.

\begin{tabular}{|c|c|c|c|c|c|c|}
\hline & \multicolumn{2}{|c|}{ Training Group (N=22) } & \multicolumn{2}{|c|}{ Control Group (N=25) } & \multirow[b]{2}{*}{$\mathbf{F}$} & \multirow[b]{2}{*}{$\mathbf{P}$} \\
\hline & $\begin{array}{l}\text { CarrierT Gln27Glu + Glu27Glu } \\
\qquad(\mathrm{N}=11)\end{array}$ & $\begin{array}{c}\text { NoncarrierT } \\
\text { Gln27Gln }(\mathrm{N}=11)\end{array}$ & $\begin{array}{c}\text { CarrierC } \\
\text { Gln27Glu }+ \\
\text { Glu27Glu }(\mathrm{N}=13)\end{array}$ & $\begin{array}{c}\text { NoncarrierC Gln27Gln } \\
\text { n (12) }\end{array}$ & & \\
\hline \multicolumn{7}{|c|}{ General characteristics and Body composition } \\
\hline Boys/girls* & $4 / 7$ & $6 / 5$ & $6 / 5$ & $8 / 4$ & 1.14 & .765 \\
\hline Age (years) & $14.7(1.21)$ & $15.0(.83)$ & $14.7(1.04)$ & $15.34(1.17)$ & .903 & .448 \\
\hline Height (m) & $1.64(.06)$ & $1.69(.08)$ & $1.66(.08)$ & $1.65(.10)$ & .607 & .614 \\
\hline Weight (kg) & $75.5(7.62)$ & $84.6(15.09)$ & $78.84(10.6)$ & $75.77(14.1)$ & 1.339 & .274 \\
\hline $\mathrm{BMI}\left(\mathrm{kg} \cdot \mathrm{m}^{2}\right)$ & $27.8(2.12)$ & $29.5(3.76)$ & $28.4(2.77)$ & $27.6(3.88)$ & .748 & .529 \\
\hline $\mathrm{WC}(\mathrm{cm})$ & $90.55(7.59)$ & $92.4(7.64)$ & $90.5(7.06)$ & 89.3 (8.97) & .295 & .829 \\
\hline FM (kg) & $28.2(4.61)$ & $30.6(10.4)$ & $32.6(10.5)$ & 30.7 (10.6) & .538 & .659 \\
\hline LM (kg & $41.5(7.17)$ & 47.5 (7.97) & $43.4(6.47)$ & $44.3(9.24)$ & 1.141 & .343 \\
\hline TFAT (kg) & $43.3(6.52)$ & $41.7(8.78)$ & $39.5(12.5)$ & $37.8(14.6)$ & .553 & .649 \\
\hline VFAT (cm) & $3.50(.71)$ & $3.59(.84)$ & $3.43(.73)$ & $3.21(.84)$ & .498 & .685 \\
\hline SFAT (cm) & $2.94(.89)$ & $3.23(.99)$ & $3.05(.93)$ & $3.37(1.23)$ & .555 & .651 \\
\hline \multicolumn{7}{|c|}{ Dietary Intake } \\
\hline TEC(kcal) & $2250.8(371.6)$ & $2263.7(481)$ & $2199.2(580)$ & $2482.6(1073)$ & .126 & .944 \\
\hline Carbohydrate(g) & $302(76.43)$ & $312(80.87)$ & 274.1(62.6) & $320.7(98,6)$ & .038 & .990 \\
\hline Proteins (g) & $85.5(21.8)$ & $96.3(25.7)$ & $91.1(34.3)$ & $90.6(31.1)$ & .398 & .755 \\
\hline Lipids(g) & $74.75(24.7)$ & $64,1(16.9)$ & $80.8(31.5)$ & $87.8(67.7)$ & .824 & .488 \\
\hline \multicolumn{7}{|c|}{ Metabolic } \\
\hline GLUC (mgddl) & $85.9(5.35)$ & $87.1(10.9)$ & $87.8(10.5)$ & $83.9(10.3)$ & .405 & .750 \\
\hline TC (mg.dl) & $165.0(42.1)$ & $169.0(46.3)$ & $164.3(13.7)$ & $157.9(43.7)$ & .173 & .914 \\
\hline
\end{tabular}




\begin{tabular}{|c|c|c|c|c|c|c|}
\hline & & & & \\
\hline LDL-c(mg.dl) & $83.1(25.2)$ & $94.6(36.5)$ & $87.9(7.89)$ & $83.5(26.9)$ & .480 & .698 \\
\hline HDL-c(mg.dl) & $59.1(14.5)$ & $55.4(11.6)$ & $56.0(8.83)$ & $54.3(14.2)$ & .316 & .814 \\
\hline VLDL (mg.dl) \# & $20.7(13.7)$ & $19.02(6.56)$ & $21.8(8.56)$ & $20.59(9.81)$ & .571 & .903 \\
\hline $\mathrm{TG}(\mathrm{mg} \cdot \mathrm{dl})^{\#}$ & $103.3(68.36)$ & $95.1(32.8)$ & $109(42.8)$ & $102.9(49.06)$ & .571 & .903 \\
\hline $\mathrm{INS}(\mu \mathrm{U} \cdot \mathrm{mL})^{\#}$ & $17.6(11.19)$ & $15.6(6.37)$ & $16.7(6.85)$ & $14.83(8.26)$ & 3.146 & .370 \\
\hline HOMA-IR ${ }^{\#}$ & $3.68(2.12)$ & $3.34(1.29)$ & $3.53(1.46)$ & $3.01(1.72)$ & 3.146 & .379 \\
\hline QUICKI & $.326(.031)$ & $.323(.018)$ & $.323(.019)$ & $.332(.020)$ & .424 & .737 \\
\hline \multicolumn{7}{|c|}{ Inflammatory markers } \\
\hline IL-6(pg.mL) & $1.51(.80)$ & $3.31(3.05)$ & $1.51(.75)$ & $1.70(.63)^{\mathrm{c}}$ & 4.431 & .009 \\
\hline $\mathrm{CPR}(\mathrm{mg} \cdot \mathrm{L})$ & $1.90(1.92)$ & $3.36(3.73)$ & $1.65(2.15)$ & $1.70(1.74)$ & .802 & .501 \\
\hline $\mathrm{TNF}-\alpha(\mathrm{pg} \cdot \mathrm{mL})^{\#}$ & $4.77(6.03)$ & $2.02(.99)$ & $1.55(1.11)$ & $5.03(5.26)$ & 5.605 & .132 \\
\hline Leptin(ng.mL $L^{\#}$ & $41.0(32.1)$ & $33.9(20.9)$ & $32.6(22.4)$ & $42.7(54.6)$ & .239 & .971 \\
\hline Resistin(ng.mL) & $9.30(3.71)$ & $8.48(3.60)$ & $8.55(4.64)$ & $7.94(1.64)$ & .358 & .784 \\
\hline $\begin{array}{l}\text { Adiponectin(ng } \\
\mathrm{mL})\end{array}$ & $9.70(7.43)$ & $7.39(4.60)^{\mathrm{d} . \mathrm{f}}$ & $7.65(3.73)$ & $6.14(3.04)^{\mathrm{a}, \mathrm{c}}$ & 23.50 & .000 \\
\hline IL-10(pg.mL) & $.313(.19)$ & $.369(.16)^{\mathrm{d} . \mathrm{f}}$ & $.306(.20)$ & $.350(.19)^{\mathrm{a} . \mathrm{c}}$ & 26.78 & .000 \\
\hline \multicolumn{7}{|c|}{ Fitness } \\
\hline $\begin{array}{l}\mathrm{VO}_{2 \max }(\mathrm{ml} . \\
\left.\mathrm{kg} \cdot{ }^{1} \min ^{-1}\right)\end{array}$ & $30.9(2.89)$ & $34.3(7.24)$ & $34.9(6.42)$ & $36.3(7.44)$ & .125 & .306 \\
\hline 1RM_Legpress & $177(35.7)$ & $183.8(61.9)$ & $176.4(35.9)$ & $182.5(53.6)$ & .067 & .977 \\
\hline$\underset{\substack{\text { 1RM_Bench } \\
\text { press }^{\#}}}{\text { 1Ren }}$ & $33.1(7.16)$ & $36.4(10.82)$ & $34.7(8.4)$ & $36.8(12.08)$ & 1.943 & .584 \\
\hline $\begin{array}{c}\text { 1RM_Arm } \\
\text { curl }\end{array}$ & $19.9(3.45)$ & $20.91(5.09)$ & $19.9(3.51)$ & $21.1(6.26)$ & .193 & .900 \\
\hline
\end{tabular}

Variable testing by chi-square, \#variable testing by Krukal-wallis. aNoncarrierC vs CarrierC; bNoncarrierC vs NoncarrierT; cNoncarrierC vs CarrierT; dCarrierC vs NoncarrierT; eCarrierC vs CarrierT; fNoncarrierT vs CarrierT. ES= Effect size; BMI= body mass index; WC= waits circumference; FM= fat mass; LM= lean mass; TFAT = trunk fat; VFAT = visceral fat; SFAT = subcutaneous fat; INS= insulin, GLUC= glucose; HOMA-IR = homeostasis model assessment; QUICKI $=$ quantitative insulin sensitivity check index; TC $=$ total cholesterol; HDL-c = high-density lipoprotein; LDL-c $=$ low-density lipoprotein; TG =triacylglycerol; $\mathrm{TNF}-\alpha=$ tumour necrosis factor-alpha; $\mathrm{IL}=$ interleukin; VO2max = maximal oxygen consumption; $1 \mathrm{RM}=$ one-repetition maximum test.

subjects who trained, those in the CarrierT group showed a higher frequency of reduced leptin levels after 12 weeks of training when compared to those in the NoncarrierT group, with a possibly beneficial effect. Both groups had reduced adiponectin and CRP levels after 12 weeks of training, although the effect size in the NoncarrierT group was possibly beneficial, while in

Table 3. Means in body composition, metabolic, inflammatory and fitness in Carrier and Noncarrier training after 12 weeks of combined training.

\section{TRAINING GROUP}

\begin{tabular}{|c|c|c|c|c|c|c|c|c|}
\hline & \multicolumn{2}{|c|}{ Gln27Glu + Glu27Glu (N=11) } & \multicolumn{2}{|c|}{ GIn27GIn $(n=11)$} & \multirow{2}{*}{$\mathbf{T}$} & \multirow{2}{*}{$\mathbf{P}$} & \multirow{2}{*}{ GXT } & \multirow{2}{*}{$\mathbf{P}$} \\
\hline & 12 weeks & $\mathbf{E S}$ & 12 weeks & ES & & & & \\
\hline \multicolumn{9}{|c|}{ General characteristics and Body composition } \\
\hline Height (m) & $1.64(.07)$ & .05 & $1.69(.08)$ & .08 & .020 & .890 & .892 & .356 \\
\hline Weight (kg) & $75.5(7.95)$ & .11 & 83.01(13.9) & .07 & .694 & .415 & .656 & .427 \\
\hline
\end{tabular}


Leite N. \& da Silva L.R. \& de Jesus Í.C. \& Lopes W.A. \& Cavaglieri C.R. \& Consentino C.L. \& Radominski R.B. \& de Souza R.L.R. \& Tureck L.V. \& Furtado-Alle L.

\begin{tabular}{|c|c|c|c|c|c|c|c|c|}
\hline BMI $\left(\mathrm{kg} / \mathrm{m}^{2}\right)$ & $27.9(2.02)$ & .14 & $28.9(3.61)$ & .27 & .298 & .591 & 1.340 & .261 \\
\hline $\mathrm{WC}(\mathrm{cm})$ & $89.6(8.37)$ & .22 & 93.9(11.7) & .21 & .013 & .911 & 1.594 & .222 \\
\hline $\mathrm{FM}(\mathrm{kg})$ & $28.1(4.60)$ & .26 & $30.6(10.4)$ & .42 & 8.688 & .008 & .459 & .506 \\
\hline $\mathrm{LM}(\mathrm{kg}$ & $43.3(7.22)$ & .16 & $48.7(7.29)$ & .24 & 82.65 & .000 & 3.329 & .083 \\
\hline TFAT (kg) & $40.6(7.16)$ & .34 & $38.5(9.74)$ & .41 & 15.467 & .001 & .056 & .816 \\
\hline VFAT (cm) & $3.29(.70)$ & .46 & $3.23(.67)$ & .29 & 6.843 & .017 & .467 & .502 \\
\hline SFAT $(\mathrm{cm})$ & $2.43(.94)$ & .46 & $2.32(.86)$ & .48 & 29.001 & .000 & .0008 & .93 \\
\hline \multicolumn{9}{|c|}{ Metabolic variables } \\
\hline GLUC (mg/dl) & $82.2(7.26)$ & .66 & $80.9(7.83)$ & .26 & 7.717 & .012 & .501 & .487 \\
\hline $\mathrm{TC}(\mathrm{mg} / \mathrm{dl})$ & 158.4(38.4) & .15 & $161.7(48.9)$ & .35 & 2.882 & .105 & .007 & .932 \\
\hline LDL-c(mg/dl) & $88.7(27.3)$ & .14 & 89.2(39.8) & .24 & .000 & .989 & 1.688 & .209 \\
\hline HDL-c(mg/dl) & $48.4(8.83)$ & .29 & 51.9(11.9) & .47 & 5.434 & .030 & 1.409 & .249 \\
\hline $\operatorname{VLDL}(\mathrm{mg} / \mathrm{dl})^{\#}$ & 21.4(11.3) & .14 & $20.6(6.62)$ & .17 & -.711 & .477 & -.711 & .477 \\
\hline $\mathrm{TG}(\mathrm{mg} / \mathrm{dl})^{\#}$ & $106.8(56.3)$ & .15 & $103.3(33.1)$ & .17 & -.800 & .424 & -.800 & .424 \\
\hline $\mathrm{INS}(\mu \mathrm{U} / \mathrm{mL})^{\#}$ & $14.3(9.73)$ & .29 & $13.6(6.91)$ & .51 & $-2.401^{\mathrm{b}}$ & .016 & - & - \\
\hline HOMA-IR ${ }^{\#}$ & 2.91(1.99) & .43 & $2.71(1.32)$ & .49 & $-2.312^{\mathrm{b}}$ & .021 & - & - \\
\hline QUICKI $^{\#}$ & $.347(.048)$ & .54 & $.335(.025)$ & .43 & $-2.045^{\mathrm{a}}$ & .041 & - & - \\
\hline \multicolumn{9}{|c|}{ Inflammatory markers } \\
\hline IL-6(pg/mL) & $1.96(1.63)$ & .56 & $1.85(1.42)$ & .54 & 4.797 & .042 & .495 & .491 \\
\hline $\mathrm{CPR}(\mathrm{mg} / \mathrm{L})$ & $1.48(2.12)$ & .21 & $1.45(1.19)$ & .39 & 10.33 & .005 & 1.876 & .189 \\
\hline $\mathrm{TNF}-\alpha(\mathrm{pg} / \mathrm{mL})$ & $6.95(8.87)$ & .24 & $1.89(1.34)$ & .15 & 2.711 & .126 & .936 & .352 \\
\hline Leptin(ng/mL) $)^{\#}$ & $41(32.08)$ & .33 & $33.87(20.9)$ & .16 & - & - & $-2.073^{b}$ & .038 \\
\hline Resistin(ng/mL) & $10.39(6.54)$ & .17 & $9.21(2.44)$ & .39 & .105 & .750 & .105 & .750 \\
\hline Adiponectin(ng/mL) & $9.70(7.43)$ & .49 & $7.39(4.60)$ & .26 & 5.615 & .029 & .200 & .660 \\
\hline IL-10(pg/mL) & $.27(.22)$ & .27 & $.49(0.77)$ & .06 & .000 & .986 & .052 & .986 \\
\hline \multicolumn{9}{|c|}{ Fitness } \\
\hline $\mathrm{VO}_{2 \max }\left(\mathrm{ml} \cdot \mathrm{kg} \cdot{ }^{1} \mathrm{~min}^{-1}\right)$ & $37.9(5.31)$ & .63 & $37.4(9.56)$ & .27 & 34.943 & .000 & 4.621 & .057 \\
\hline 1RM_Legpress ${ }^{\#}$ & 240.3(42.9) & .63 & $200.8(36.7)$ & .84 & $-2.388^{b}$ & .017 & - & - \\
\hline 1RM_Bench press & $46.6(10.8)$ & .63 & 38.4(8.08) & .63 & 77.231 & .000 & 3.782 & .078 \\
\hline 1RM_Arm curl & 23.7(4.19) & .74 & $23(3.84)$ & .87 & 11.260 & .006 & .594 & .457 \\
\hline
\end{tabular}

Values expressed as mean (standard deviation); \#variables testing by Wilcoxon-signed Rank. GXT= group*time; aranks negativos; branks positivos. ES= Effect size; $\mathrm{BMI}=$ body mass index; $\mathrm{WC}=$ waits circumference; $\mathrm{FM}=$ fat mass; $\mathrm{LM}=$ lean mass; $\mathrm{TFAT}=$ trunk fat; $\mathrm{VFAT}=$ visceral fat; $\mathrm{SFAT}=$ subcutaneous fat; $\mathrm{TEC}=$ total energy consumption; INS = insulin, HOMA-IR = homeostasis model assessment; QUICKI = quantitative insulin sensitivity check index; TC = total cholesterol; HDL-c = high-density lipoprotein; LDL-c = low-density lipoprotein; TG=triacylglycerol; TNF- $\alpha=$ tumour necrosis factor-alpha; IL = interleukin; VO2max = maximal oxygen consumption $1 \mathrm{RM}=$ one-repetition maximum test.

the Carrier group it was unclear for adiponectin and probably trivial CRP (Figure 2).

After 12 weeks, the CarrierC group showed an increase in height, which was not observed in the training group. Both carrier groups showed an increase in WC, although the effect size was greater in the group that did not train $(\mathrm{ES}=.74 \mathrm{vs} \mathrm{ES}=.22)$. No main effect or interactions between CarrierT and CarrierC groups were shown in TEC $(\mathrm{F}=1.938 ; \mathrm{p}=.189)$; carbohydrates $(\mathrm{F}=.158$; $\mathrm{p}=.698)$, proteins $(\mathrm{F}=1.960 ; \mathrm{p}=.187)$ and lipids $(\mathrm{F}=.571 ; \mathrm{p}=.464)$.

The participants of the CarrierT group showed a reduction 
A

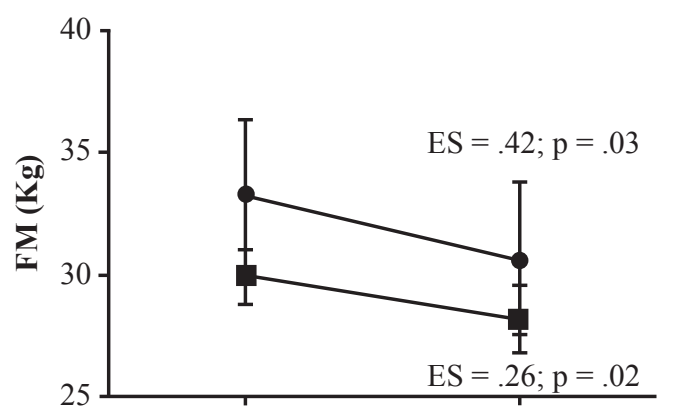

C

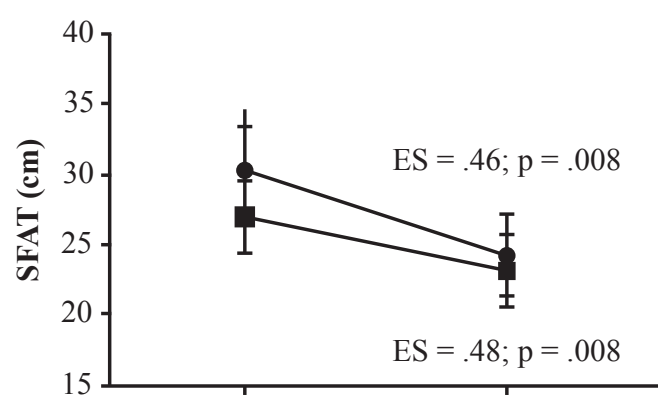

$\mathbf{E}$

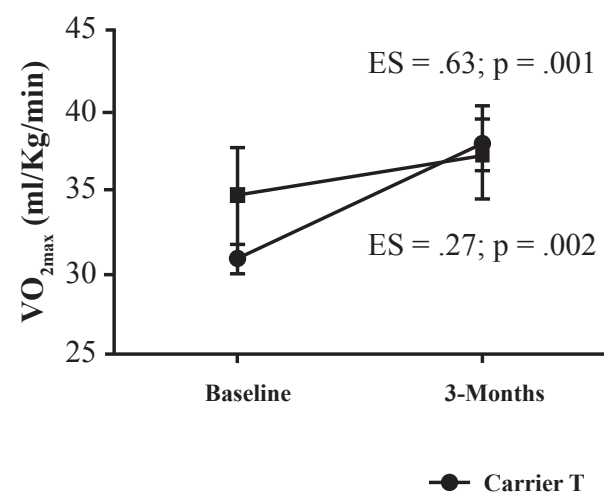

B

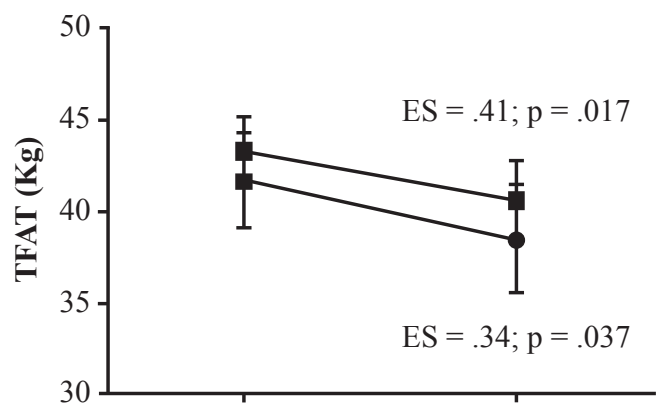

D

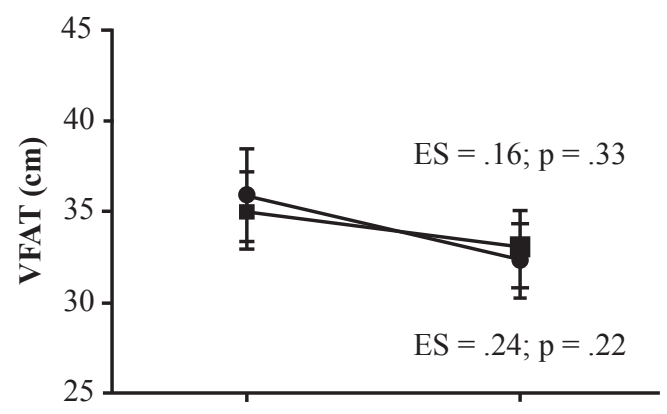

F

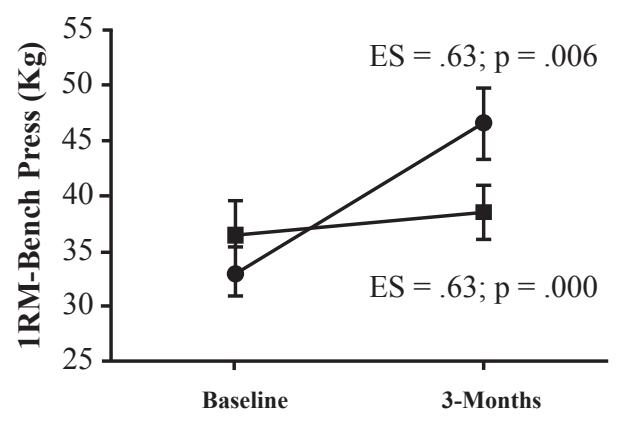

Noncarrier T

Figure 1. Means of Fat Mass (FM) (A), Trunk Fat (TFAT) (B), Subcutaneous Fat (SFAT) (C), Visceral Fat (VFAT) (D), maximal oxygen consumption (VO2max) (E) and 1-RM Bench Press (F) in the Carrier and Noncarrier Training groups at baseline and after 3 months of training. Note: Carrier $\mathrm{T}=\mathrm{Gln} 27 \mathrm{Glu}$ or Glu27Glu; NoncarrierT $=\mathrm{Gln} 27 \mathrm{Gln}$; ES= Effect size.

of FM and TFAT, as well as an increase in $\mathrm{VO}_{2 \max }$, and in the leg press and arm curl test values. In the CarrierC group, after the 12 weeks of training, most participants showed lower strength test values and higher HOMA-IR values than the CarrierT group (Table 3). Participants of both Carrier groups had a reduction in subcutaneous abdominal fat. However, the effect size was greater in the group that trained $(\mathrm{ES}=.48$ vs $\mathrm{ES}=.17)$. As for the inflammatory variables, while the CarrierT group showed a reduction in leptin levels after 12 weeks of training, the CarrierC group showed an increase of such levels.

After the intervention, the NoncarrierC group showed an increase in height and in WC, which was not observed in the NoncarrierT group. The participants in the NoncarrierT group showed a reduction in TFAT, as well as an increase in LM, LEG press and bench press, while the NoncarrierC group did not. No main effect or interactions between NoncarrierT and NonarrierC groups were shown in TEC $(\mathrm{F}=1.314 ; \mathrm{p}=.269)$, carbohydrates $(\mathrm{F}=2.244 ; \mathrm{p}=.154)$, proteins $(\mathrm{F}=1.218 ; \mathrm{p}=.285)$ and lipids $\left(\mathrm{Z}=-1.540^{\mathrm{b}} ; \mathrm{p}=.123\right)$.

When compared to the control group, the NoncarrierT group had a lower frequency of subjects who showed an increase in insulin levels, as well as a higher frequency of subjects with reduced HOMA-IR values and increased QUICKI values after the 12 weeks of training. The NoncarrierT group had a higher frequency of subjects with increased $\mathrm{VO}_{2 \max }$ after the 12 weeks than the NoncarrierC group. The NoncarrierC showed a reduction in LDL-c values, while the training group did not. The NoncarrierT group showed a reduction in HDL-c values after the 12 weeks of training, which was not observed in the training group. 
Leite N. \& da Silva L.R. \& de Jesus Í.C. \& Lopes W.A. \& Cavaglieri C.R. \& Consentino C.L. \& Radominski R.B. \& de Souza R.L.R. \& Tureck L.V. \& Furtado-Alle L.

A

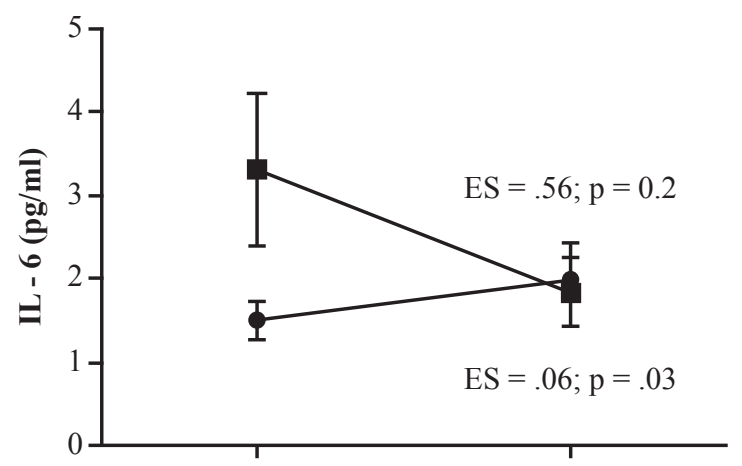

$\mathrm{C}$

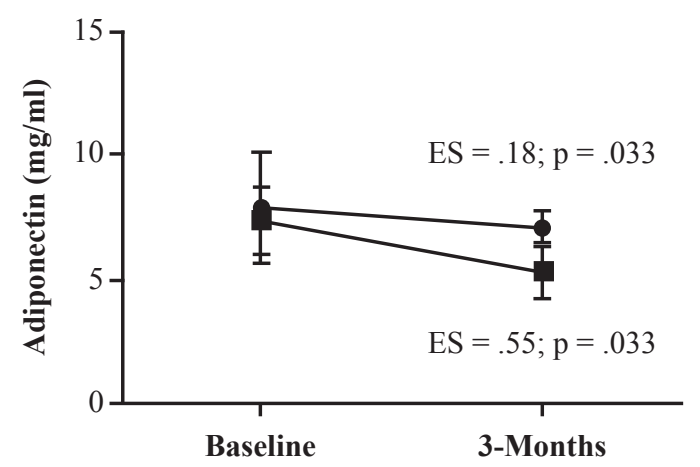

B

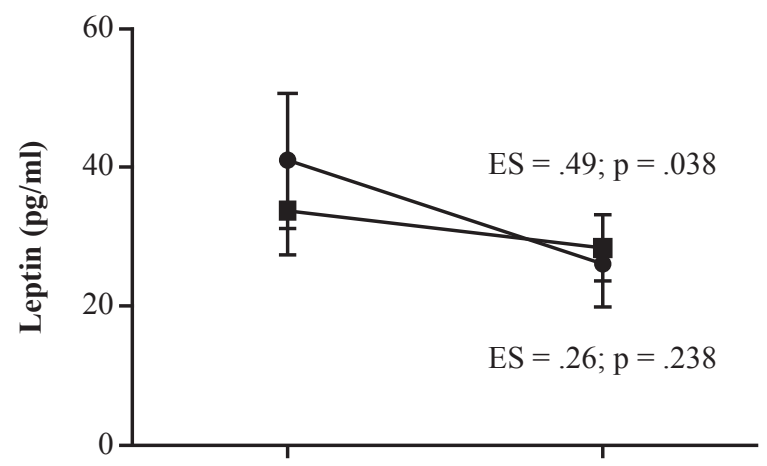

$\mathrm{D}$

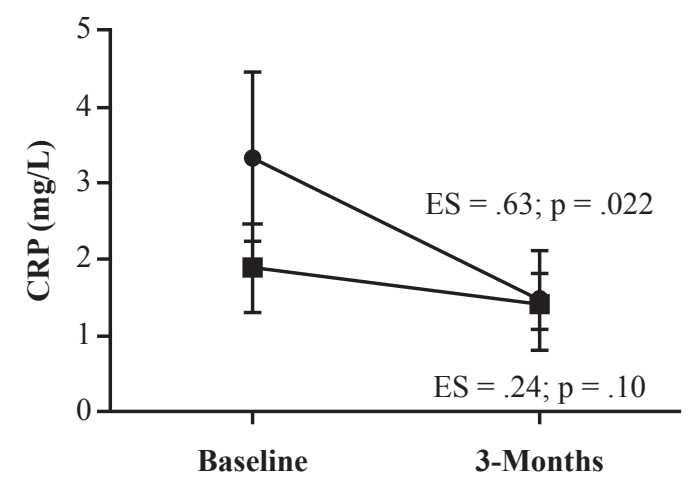

Carrier T

Noncarrier T

Figure 2. Means of Interleukin-6 (IL-6) (A), Leptin (B), Adiponectin (C), C-reative Protein (CRP) (D) in the Carrier and in the Noncarrier Training groups at baseline and after 3 months of training. Note: CarrierT $=$ Gln27Glu or Glu27Glu; NoncarrierT= Gln27Gln; ES= Effect size.

Table 4. Changes in body composition, metabolic, inflammatory and fitness in Carrier and Noncarrier after 12 weeks of combined training.

\begin{tabular}{|c|c|c|c|c|c|c|c|c|c|c|c|c|c|c|c|c|}
\hline \multicolumn{9}{|c|}{ Gln27Gln } & \multicolumn{8}{|c|}{ GIn27Glu + Glu27Glu } \\
\hline & \multicolumn{2}{|c|}{$\begin{array}{c}\text { NoncarrierT } \\
(\mathbf{N}=11)\end{array}$} & \multicolumn{2}{|c|}{$\begin{array}{c}\text { NoncarrierC } \\
(\mathbf{N}=12)\end{array}$} & \multirow[t]{2}{*}{$\mathbf{T}$} & \multirow[t]{2}{*}{$\mathbf{P}$} & \multirow[t]{2}{*}{ GXT } & \multirow[t]{2}{*}{$\mathbf{P}$} & \multicolumn{2}{|c|}{$\begin{array}{c}\text { CarrierT } \\
(\mathrm{n}=11)\end{array}$} & \multicolumn{2}{|c|}{$\begin{array}{c}\text { CarrierC ( } \\
\mathrm{N}=13)\end{array}$} & \multirow[t]{2}{*}{$\mathbf{T}$} & \multirow[t]{2}{*}{$\mathbf{P}$} & \multirow[t]{2}{*}{ GXT } & \multirow[t]{2}{*}{$\mathbf{P}$} \\
\hline & Change & ES & Change & ES & & & & & Change & ES & Change & ES & & & & \\
\hline \multicolumn{17}{|c|}{ General characteristics and Body composition } \\
\hline $\begin{array}{l}\text { Height } \\
\text { (m) }\end{array}$ & $\begin{array}{c}.003 \\
(.023)\end{array}$ & .08 & $\begin{array}{l}.018 \\
(.012)\end{array}$ & .60 & 3.994 & .059 & 7.657 & .012 & $\begin{array}{l}.004 \\
(.009)\end{array}$ & .05 & $\begin{array}{c}.012 \\
(.009)\end{array}$ & .12 & 17.408 & .000 & 4.506 & .045 \\
\hline $\begin{array}{l}\text { Weight } \\
(\mathrm{kg})\end{array}$ & $\begin{array}{c}-.02 \\
(2.15)\end{array}$ & .07 & $\begin{array}{l}1.85 \\
(2.37)\end{array}$ & .17 & 3.578 & .073 & 3.758 & .067 & $\begin{array}{l}-1.60 \\
(6.09)\end{array}$ & .11 & $\begin{array}{c}3.51 \\
(6.35)\end{array}$ & .25 & .559 & .463 & 4.006 & .058 \\
\hline $\begin{array}{c}\text { BMI } \\
(\mathrm{kg} \cdot \mathrm{m} 2)\end{array}$ & $.18(.04)$ & .27 & $\begin{array}{l}.51 \\
(.81)\end{array}$ & .40 & 5.173 & .034 & 1.121 & .302 & $\begin{array}{c}-.51 \\
(1.91)\end{array}$ & .14 & $\begin{array}{c}1.01 \\
(1.97)\end{array}$ & .29 & .384 & .542 & 3.660 & .069 \\
\hline $\begin{array}{l}\text { WC } \\
(\mathrm{cm})\end{array}$ & $\begin{array}{l}-1.86 \\
(4.86)\end{array}$ & .21 & $\begin{array}{l}2.97 \\
(3.86)\end{array}$ & .38 & .344 & .564 & 6.421 & .028 & $\begin{array}{l}1.55 \\
(7.15)\end{array}$ & .22 & $\begin{array}{c}3.64 \\
(3.48)\end{array}$ & .74 & 5.038 & .036 & .817 & .376 \\
\hline $\begin{array}{l}\text { FM } \\
(\mathrm{kg})\end{array}$ & $\begin{array}{l}-1.69 \\
(2.58)\end{array}$ & .45 & $\begin{array}{c}.45 \\
(1.35)\end{array}$ & .31 & 1.350 & .262 & 4.064 & .061 & $\begin{array}{l}-2.70 \\
(4.22)\end{array}$ & .27 & $\begin{array}{c}.65 \\
(1.70)\end{array}$ & .06 & 1.671 & .213 & 4.468 & .050 \\
\hline $\begin{array}{l}\mathrm{LM} \\
(\mathrm{kg})\end{array}$ & $\begin{array}{c}1.76 \\
(1.35)\end{array}$ & .58 & $\begin{array}{c}-.04 \\
(2.15)\end{array}$ & .04 & 4.380 & .053 & 4.822 & .043 & $\begin{array}{c}1.19 \\
(1.64)\end{array}$ & .60 & $\begin{array}{l}.32 \\
(1.06)\end{array}$ & .30 & 5.162 & .036 & 1.703 & .209 \\
\hline $\begin{array}{l}\text { TFAT } \\
(\mathrm{kg})\end{array}$ & $\begin{array}{l}-2.78 \\
(3.22)\end{array}$ & .47 & $\begin{array}{c}.65 \\
(2.53)\end{array}$ & .20 & 2.168 & .163 & 5.681 & .030 & $\begin{array}{l}-3.13 \\
(3.81)\end{array}$ & .34 & $\begin{array}{c}.62 \\
(2.10)\end{array}$ & .07 & 2.817 & .112 & 6.319 & .022 \\
\hline
\end{tabular}




\begin{tabular}{|c|c|c|c|c|c|c|c|c|c|c|c|c|c|c|c|c|}
\hline $\begin{array}{l}\text { VFAT } \\
(\mathrm{cm})\end{array}$ & $\begin{array}{l}-.21 \\
(.53)\end{array}$ & .20 & $\begin{array}{l}-.26 \\
(.47)\end{array}$ & .32 & 4.710 & .042 & .056 & .816 & $\begin{array}{l}-.35 \\
(.47)\end{array}$ & .46 & $\begin{array}{l}.026 \\
(.67)\end{array}$ & .04 & 1.820 & .191 & 2.451 & .132 \\
\hline $\begin{array}{r}\text { SFAT } \\
(\mathrm{cm})\end{array}$ & $\begin{array}{l}-.42 \\
(.33)\end{array}$ & .48 & $\begin{array}{l}.021 \\
(.62)\end{array}$ & .02 & 3.286 & .087 & 4.010 & .061 & $\begin{array}{c}-44 \\
(.38)\end{array}$ & .46 & $\begin{array}{l}-17 \\
(.44)\end{array}$ & .17 & 11.838 & .003 & 2.160 & .157 \\
\hline \multicolumn{17}{|c|}{ Metabolic } \\
\hline $\begin{array}{c}\text { GLUC } \\
\text { (mg.dl) }\end{array}$ & $\begin{array}{l}-3.68 \\
(9.12)\end{array}$ & .26 & $\begin{array}{l}-5.60 \\
(10.8)\end{array}$ & .34 & 4.712 & .042 & .202 & .658 & $\begin{array}{l}-6.20 \\
(7.48)\end{array}$ & .66 & $\begin{array}{l}-3.02 \\
(5.42)\end{array}$ & .29 & 12.226 & .002 & 1.443 & .242 \\
\hline $\begin{array}{c}\mathrm{TC} \\
\text { (mg.dl) }\end{array}$ & $\begin{array}{c}-6.60 \\
(15.48)\end{array}$ & .35 & $\begin{array}{c}-9.51 \\
(13.31)\end{array}$ & .45 & 6.846 & .017 & .224 & .641 & $\begin{array}{c}-7.31 \\
(22.32)\end{array}$ & .15 & $\begin{array}{c}.39 \\
(19.82)\end{array}$ & .00 & .646 & .430 & .802 & .380 \\
\hline $\begin{array}{l}\text { LDL-c } \\
\text { (mg.dl) }\end{array}$ & $\begin{array}{c}5.53 \\
(12.44)\end{array}$ & .24 & $\begin{array}{c}-13.27 \\
(17.04)\end{array}$ & .47 & 1.478 & .238 & 8.742 & .008 & $\begin{array}{c}-5.41 \\
(25.05)\end{array}$ & .14 & $\begin{array}{c}-.73 \\
(28.07)\end{array}$ & .19 & .316 & .580 & .183 & .673 \\
\hline $\begin{array}{l}\text { HDL-c } \\
\text { (mg.dl) }\end{array}$ & $\begin{array}{l}-10.7 \\
(12.4)\end{array}$ & .47 & $\begin{array}{c}1.89 \\
(13.8)\end{array}$ & .07 & 2.483 & .036 & 5.071 & .036 & $\begin{array}{c}-3.49 \\
(15.95)\end{array}$ & .29 & $\begin{array}{c}2.02 \\
(18.06)\end{array}$ & .13 & .044 & .837 & .613 & .440 \\
\hline $\begin{array}{l}\text { VLDL } \\
\text { (mg.dl) \# }\end{array}$ & $\begin{array}{l}-1.40 \\
(7.75)\end{array}$ & .17 & $\begin{array}{c}1.87 \\
(8.18)\end{array}$ & .30 & - & - & -.800 & .424 & $\begin{array}{c}1.62 \\
(6.98)\end{array}$ & .14 & $\begin{array}{c}-.89 \\
(7.19)\end{array}$ & .00 & - & - & -.711 & .477 \\
\hline $\begin{array}{c}\text { TG(mg. } \\
\text { dl) \# }\end{array}$ & $\begin{array}{l}-7.01 \\
(38.8)\end{array}$ & .17 & $\begin{array}{c}9.32 \\
(40.93)\end{array}$ & .30 & - & - & -.800 & .424 & $\begin{array}{c}8.17 \\
(34.9)\end{array}$ & .15 & $\begin{array}{l}-4.46 \\
(35.9)\end{array}$ & .00 & - & - & -.711 & .477 \\
\hline $\begin{array}{c}\mathrm{INS}(\mu \mathrm{U} . \\
\mathrm{mL}) \#\end{array}$ & $\begin{array}{l}-4.00 \\
(4.18)\end{array}$ & .51 & $\begin{array}{c}3.51 \\
(4.85)\end{array}$ & .39 & - & - & $-2.401 b$ & .016 & $\begin{array}{l}-2.05 \\
(4.17)\end{array}$ & .29 & $\begin{array}{c}1.03 \\
(5.93)\end{array}$ & .07 & - & - & -1.379 & .701 \\
\hline $\begin{array}{l}\text { HOMA- } \\
\text { IR\# }\end{array}$ & $\begin{array}{l}-0.95 \\
(1.09)\end{array}$ & .49 & $\begin{array}{l}.52 \\
(.92)\end{array}$ & .36 & - & - & $-2.312 \mathrm{a}$ & .021 & $\begin{array}{l}-.62 \\
(.86)\end{array}$ & .43 & $\begin{array}{c}.09 \\
(1.23)\end{array}$ & .04 & - & - & $-2.045 b$ & .041 \\
\hline QUICKI\# & $\begin{array}{l}.021 \\
(.31)\end{array}$ & .43 & $\begin{array}{l}-.009 \\
(.016)\end{array}$ & .28 & - & - & $-2.045 b$ & .041 & $\begin{array}{l}.012 \\
(.017)\end{array}$ & .54 & $\begin{array}{c}-.001 \\
(.021)\end{array}$ & .04 & 1.869 & .185 & 2.563 & .124 \\
\hline \multicolumn{17}{|c|}{ Inflammatory markers } \\
\hline $\begin{array}{c}\text { IL-6 } \\
\text { (pg.mL) }\end{array}$ & $\begin{array}{l}-1.72 \\
(3.09)\end{array}$ & .56 & $\begin{array}{c}.25 \\
(1.11)\end{array}$ & .44 & .319 & .581 & 1.997 & .179 & $\begin{array}{c}.32 \\
(1.57)\end{array}$ & .06 & $\begin{array}{c}-.24 \\
(1.31)\end{array}$ & .52 & 1.324 & .267 & .741 & .402 \\
\hline $\begin{array}{c}\text { CPR } \\
\text { (mg.L) }\end{array}$ & $\begin{array}{c}-2.02 \\
(3.40)\end{array}$ & .63 & $\begin{array}{c}.28 \\
(1.23)\end{array}$ & .19 & 1.093 & .312 & 3.598 & .077 & $\begin{array}{l}-.11 \\
(.94)\end{array}$ & .24 & $\begin{array}{c}-.56 \\
(2.05)\end{array}$ & .14 & .822 & .378 & .069 & .796 \\
\hline $\begin{array}{c}\text { TNF- } \alpha \\
\text { (pg.mL)\# }\end{array}$ & $\begin{array}{c}.27 \\
(1.32)\end{array}$ & .09 & $\begin{array}{l}-.55 \\
(6.54)\end{array}$ & .00 & - & - & -.338 & .735 & $\begin{array}{c}1.66 \\
(1.93)\end{array}$ & .24 & $\begin{array}{c}.14 \\
(1.29)\end{array}$ & .31 & 2.649 & .124 & .170 & .686 \\
\hline $\begin{array}{l}\text { Leptin(ng. } \\
\text { mL\# }\end{array}$ & $\begin{array}{c}-5.54 \\
(14.36)\end{array}$ & .26 & $\begin{array}{c}1.81 \\
(12.12)\end{array}$ & .03 & - & - & -1.245 & .213 & $\begin{array}{l}-7.80 \\
(9.16)\end{array}$ & .49 & $\begin{array}{c}10.83 \\
(14.76)\end{array}$ & .41 & .422 & .524 & 8.781 & .008 \\
\hline $\begin{array}{l}\text { Resistin } \\
\text { (ng.mL) }\end{array}$ & $\begin{array}{c}.73 \\
(2.80)\end{array}$ & .39 & $\begin{array}{c}.71 \\
(3.08)\end{array}$ & .27 & 1.712 & .208 & .108 & .746 & $\begin{array}{c}2.12 \\
(3.15)\end{array}$ & .41 & $\begin{array}{c}.71 \\
(3.16)\end{array}$ & .30 & 4.216 & .054 & 1.058 & .317 \\
\hline $\begin{array}{c}\text { Adipo- } \\
\text { nectin } \\
\text { (ng.mL) }\end{array}$ & $\begin{array}{c}-2.00 \\
(5.23)\end{array}$ & .55 & $\begin{array}{c}.08 \\
(3.15)\end{array}$ & .03 & 1.277 & .274 & 1.599 & .223 & $\begin{array}{c}-.79 \\
(2.34)\end{array}$ & .18 & $\begin{array}{c}-.27 \\
(2.82)\end{array}$ & .06 & .612 & .44 & .063 & .805 \\
\hline $\begin{array}{c}\text { IL-10 } \\
\text { (pg.mL) }\end{array}$ & $\begin{array}{l}.195 \\
(.81)\end{array}$ & .06 & $\begin{array}{l}.018 \\
(.21)\end{array}$ & .08 & .001 & .972 & .047 & .832 & $\begin{array}{l}-.045 \\
(.27)\end{array}$ & .28 & $\begin{array}{l}.041 \\
(.15)\end{array}$ & .12 & .002 & .964 & .783 & .387 \\
\hline \multicolumn{17}{|c|}{ Fitness } \\
\hline $\begin{array}{l}\text { VO2max } \\
(\mathrm{ml} . \mathrm{kg} . \\
\text { 1min.-1)\# }\end{array}$ & $\begin{array}{c}5.57 \\
(2.86)\end{array}$ & .63 & $\begin{array}{c}1.21 \\
(4.19)\end{array}$ & .00 & - & - & $-2.371 b$ & .018 & $\begin{array}{c}2.60 \\
(1.28)\end{array}$ & .27 & $\begin{array}{c}-.56 \\
(2.13)\end{array}$ & .08 & 3.635 & .083 & 8.828 & .013 \\
\hline $\begin{array}{l}\text { 1RM_- } \\
\text { Legpress }\end{array}$ & $\begin{array}{c}53.1 \\
(23.6)\end{array}$ & .63 & $\begin{array}{c}15.7 \\
(13.04)\end{array}$ & .54 & 45.524 & .000 & 13.451 & .003 & $\begin{array}{c}56.67 \\
(39.83)\end{array}$ & .84 & $\begin{array}{c}10.9 \\
(12.61)\end{array}$ & .20 & 27.921 & .000 & 12.802 & .003 \\
\hline $\begin{array}{l}1 \mathrm{RM} \\
\text { Bench } \\
\text { press }\end{array}$ & $\begin{array}{c}12.3 \\
(4.07)\end{array}$ & .51 & $\begin{array}{c}2.71 \\
(2.62)\end{array}$ & .54 & 67.089 & .000 & 27.316 & .000 & $\begin{array}{c}7.83 \\
(4.16)\end{array}$ & .63 & $\begin{array}{c}.54 \\
(2.91)\end{array}$ & .14 & - & - & $-2.207 \mathrm{a}$ & .027 \\
\hline $\begin{array}{l}\text { 1RM_ } \\
\text { Arm curl }\end{array}$ & $\begin{array}{c}2.71 \\
(4.53)\end{array}$ & .74 & $\begin{array}{l}1.42 \\
(1.39)\end{array}$ & .54 & 5.334 & .040 & .514 & .487 & $\begin{array}{c}4.33 \\
(2.58)\end{array}$ & .87 & $\begin{array}{c}1.45 \\
(2.38)\end{array}$ & .20 & - & - & 5.359 & .035 \\
\hline
\end{tabular}

Values expressed as mean (standard deviation); \# variables testing by Wilcoxon-signed Rank. anegative ranks; bpositive ranks. ES= Effect size; $\mathrm{BMI}=$ body mass index; $\mathrm{WC}=$ waits circumference; $\mathrm{FM}=$ fat mass; $\mathrm{LM}=$ lean mass; $\mathrm{TFAT}=$ trunk fat; VFAT $=$ visceral fat; $\mathrm{SFAT}=$ subcutaneous fat; $\mathrm{TEC}=$ total energy consumption; INS = insulin, HOMA-IR = homeostasis model assessment; QUICKI = quantitative insulin sensitivity check index; TC $=$ total cholesterol; HDL-c $=$ high-density lipoprotein; LDL-c $=$ low-density lipoprotein; TG=triacylglycerol; TNF- $\alpha=$ tumour necrosis factor-alpha; $\mathrm{IL}=$ interleukin; $\mathrm{VO} 2 \mathrm{max}=$ maximal oxygen consumption $1 \mathrm{RM}=$ one-repetition maximum test. GxT= Group*Time 


\section{Discussion}

This study investigated the influence of $A D R B 2$ Gln27Glu polymorphism in anthropometric, metabolic and inflammatory parameters in overweight adolescents after twelve weeks of combined training (CT). The CT was effective in reducing total fat mass, subcutaneous abdominal fat, increasing lean mass, and fitness independent of presence of $A D R B 2$ Gln27Glu polymorphism. However, only the Glu27 allele carriers showed reductions in leptin after 12-weeks.

At baseline, Glu27carriers and noncarriers had similar body composition, physical fitness, anthropometric and metabolic parameters. These results are in line with other studies with children and adolescents that found no differences in anthropometric ${ }^{34,35}$, metabolic and cardiorespiratory fitness parameter ${ }^{36}$ or risk of bronchospasm due to exercise ${ }^{37}$ between carriers and noncarriers. Conversely, other studies indicate that the Glu27 allele is associated with greater body mass ${ }^{36}$, body mass index ${ }^{38}$, waist circumference $^{39}$, and higher blood pressure and triglycerides levels ${ }^{40}$, when compared to Glu27 allele noncarriers. On the other hand, higher total cholesterol levels have been found in adolescents homozygotes for the usual allele (Gln27Gln) when compared to carriers of the Glu27 variant ${ }^{36}$. With regard to the presence or absence of the Glu27 allele in groups whose leptin, CRP, TNF-alpha and resistin levels, they were similar at baseline. In contrast, all the four groups presented differences in adiponectin and IL-6 levels. This result, however, was independent of the presence of the Glu27allele. Carrier adolescents had lower IL-10 levels than noncarrier adolescents, which suggests that they have a lower protective effect, since IL-10 is a cytokine with anti-inflammatory properties that plays a central role in infections by controlling immune responses to pathogens ${ }^{41}$.

After 12 weeks of training, our results have shown that Glu27 allele carriers who trained had a reduction in total fat mass and trunk fat mass, and an increase in lean body mass, but no changes in body mass, BMI and WC. The effect size was greater in the Glu27 allele carrier group who trained, when analyzing the reduction in total fat mass $(\mathrm{ES}=.46$ vs $\mathrm{ES}=.26$ ) and trunk fat mass $(\mathrm{ES}=.41 \mathrm{vs} \mathrm{ES}=.34)$. Our results disagree with another study conducted with children and adolescents, in which a reduction in weight, BMI z-score and WC was identified in ADRB2 Glu27 carriers and noncarriers after 12 weeks of aerobic exercises ${ }^{40}$. This divergence is probably due to the fact that the adolescents in this study undertook a lower volume of aerobic physical activity and exhibited an increase in lean body mass as a result of resistance exercises. Similarly, in a study with postmenopausal women, in which intervention consisted of 12 months of resistance training, carriers showed a greater increase in lean body mass than noncarriers, even though no significant changes were found in body composition ${ }^{43}$.

As for the metabolic parameters, Glu27 carriers exhibited reductions only in blood glucose levels after the 12 weeks of training. The effect, however, was probably beneficial only for the group that trained, whereas the control group only experienced a trivial effect. In addition, carriers who undertook the intervention had a higher frequency of improvement of IR than controls, which suggests that the performance of this kind of exercise protocol by adolescents, regardless of the genotype of the GIn27Glu polymorphism, may help control and prevent the progression of glucose metabolism-related disorders.

Although no significant difference was found after the 12 weeks of training, the intervention groups exhibited changed levels of IL-6, leptin, adiponectin and CRP. The NoncarrierT group had reduced IL-6 levels after the 12 weeks of training, showing a probably beneficial effect. This may be associated with greater IL-6 levels at baseline, as well as with reduced CRP levels after combined training. Considering that IL-6 has been described as being a cytokine with both pro- and anti-inflammatory properties ${ }^{44}$, the reduction in IL-6 and CRP levels may indicate a decrease in the inflammatory process in the NoncarrierT group. Exercise may have both a direct antiinflammatory effect, through an increase in the production of anti-inflammatory cytokines such as IL-6 by the skeletal muscle, and an indirect anti-inflammatory effect, through the reduction of adipose tissue, which results in a lower release of cytokines ${ }^{45}$.

After 12 weeks of CT, only the individuals in the CarrierT group had lower levels of circulating leptin (ES=.49), showing better response in reducing body fat through the performance of this training program than the individuals in the NoncarrierT group. Leptin stimulates glucose uptake through activation of adenosine monophosphate-activated protein kinase (AMPK). However, at high concentrations, it may inhibit AMPK activation, because it increases insulin receptor substrate-1 expression, due to the activation of the suppressor of cytokine signalling $3^{46}$. In the present study, these effects may be associated with the reductions in FM observed after the intervention, which consequently led to an improvement of the inflammatory status and of insulin response. Moreover, individuals in the CarrierC group showed increased leptin levels after 12 weeks of training, with a moderate effect size (ES=.41), which suggests that inactivity may be another obesogenic factor, whereas regular physical exercise probably promotes reductions and improvements in the inflammatory profile. In adults, the homozygous Glu27 genotype ${ }^{47}$ appears to be associated with greater leptin concentrations than the other two genotypes.

With regards to physical fitness, both groups showed increased $\mathrm{VO}_{2 \max }$ and $1 \mathrm{RM}$ bench press values. Nevertheless, the effect size regarding $\mathrm{VO}_{2 \max }$ increase was greater in the CarrierT group ( $\mathrm{ES}=.63$ vs $\mathrm{ES}=.27$; possibly beneficial) than in the NoncarrierT group. All groups showed changes in lean body mass. Nevertheless, when compared to their controls, both carriers and noncarriers exhibited probably beneficial effects, which suggests that training was effective in increasing lean body mass, regardless of the $\beta 2$-adrenergic receptors.

The lack of intervention, especially in the Glu27allele carrier group, led to a significant increase in waist circumference with a great effect size, while the magnitude of effect was smaller in the NoncarrierC group. Therefore, the lack of training in Glu27 allele carriers have deleterious effects on abdominal adiposity, as well as an increase in leptin levels in this group, which was not observed in any of the other groups. Beta-adrenergic receptors are one of the main regulators of lipolysis, controlling the activation of intracellular AMP-c levels and thereby increasing the protein kinase A response, which stimulates hormone-sensitive lipase 
activity, resulting in the release of circulating free fatty acids ${ }^{48}$. Considering that, $A D R B 2$ Gln27Glu polymorphism seems to result in reduced receptor sensibility, which leads to a reduction in lipase activity ${ }^{49}$. It may be suggested that regular physical exercise acts as a compensatory and mitigating mechanism in the change generated by the presence of the Glu27 allele, since exercise can stimulate sympathetic nervous system activity, thus stimulating lipolysis.

One limitation of this study is the small number of participants, which prevents the performance of a gender-specific analysis. However, all participants were in the postpubertal stage, in which the morphological and biological differences between genders are milder than in puberty ${ }^{50}$. Moreover, the analyses and comparisons made were limited to the Gln27Glu polymorphism, other variants of the beta adrenergic genes have not been assessed. Findings of previous studies indicate the existence of links between variants which, when combined, increase the risk of and the susceptibility to obesity ${ }^{48}$. In view of this, further research is needed taking into consideration the other polymorphisms of the ADRB2 gene. Besides, further research including different exercise modalities and intensities could improve the understanding of interactions between variants of the ADRB2 gene and adolescents' responsiveness to exercise.

We conclude that CT led to changes in body composition, regardless of the genotypes of the $A D R B 2$ Gln27Glu polymorphism. Moreover, the presence of the Glu27 allele did not result in a negative effect, since Glu27 carriers responded better to training than noncarriers. This was shown by a greater effect size on reduction in body composition, as well as by an increase in fitness. Nevertheless, only the Glu27 allele carriers showed reductions in leptin levels after 12 weeks. Another important factor is that the Glu27 allele carriers had lower IL-10 levels at baseline, which gives them a lower anti-inflammatory protection. Lack of regular physical activity in overweight adolescents, especially in the Glu27 carrier group, was associated with increased obesity indicators and leptin levels in adolescents, which suggests that exercise can be an important tool to mitigate the changes associated with possible risk alleles.

\section{References}

1. MacPherson M, de Groh M, Loukine L, Prud'homme D, Dubois L. Prevalence of metabolic syndrome and its risk factors in Canadian children and adolescents: Canadian Health Measures Survey Cycle 1 (2007-2009) and Cycle 2 (2009-2011). Heal Promot chronic Dis Prev Canada Res policy Pract. 2016;36(2):3240. http://www.ncbi.nlm.nih.gov/pubmed/26878492. Accessed June 2, 2016.

2. Silva LR, Stefanello JMF, Pizzi J, Timossi LS, Leite N. Aterosclerose subclínica e marcadores inflamatórios em crianças e adolescentes obesos e não obesos. Rev Bras Epidemiol. 2012;15(4):804-816. doi:10.1590/S1415-790X2012000400012.

3. Nemet D, Barkan S, Epstein Y, Friedland O, Kowen G, Eliakim A. Short- and long-term beneficial effects of a combined dietarybehavioral-physical activity intervention for the treatment of childhood obesity. Pediatrics. 2005;115(4):e443-9. doi:10.1542/ peds.2004-2172.

4. Miles L. Physical activity and health. Nutr Bull. 2007;32:314-363. doi:10.1111/j.1467-3010.2007.00668.x.

5. Moreno-Aliaga MJ, Santos JL, Marti A, Martínez JA. Does weight loss prognosis depend on genetic make-up? Obes Rev. 2005;6(2):155-168. doi:10.1111/j.1467-789X.2005.00180.x.

6. Chou YC, Tsai CN, Lee YS, Pei JS. Association of adrenergic receptor gene polymorphisms with adolescent obesity in Taiwan. Pediatr Int. 2012;54(1):111-116. doi:10.1111/j.1442-200X.2011.03516.x.

7. Masuo K, Rakugi H, Ogihara T. Relationships of Beta2- and Beta3--Adrenoceptor Polymorphisms with Obesity, Hypertension and Metabolic Syndrome. Curr Hypertens Rev. 2010;6(2):118129. doi:10.2174/157340210791171065.

8. Masuo K, Katsuya T, Kawaguchi H, Rakugi YFH, Ogihara T, Tuck ML. Rebound weight gain as associated with high plasma norepinephrine levels that are mediated through polymorphisms in the beta2-adrenoceptor. Am J Hypertens. 2005;18(11):15081516. doi:10.1016/j.amjhyper.2005.05.006.

9. Villares SM, Mancini MC, Gomez S, Charf AM, Frazzatto E, Halpern A. Associação entre polimorfismo Gln27Glu do receptor beta2-adrenérgico e hipertensão arterial sistêmica em obesos mórbidos. Arq Bras Endocrinol Metabol. 2000;44(1):72-80. doi:10.1590/S0004-27302000000100012.

10. Masuo K, Katsuya T, Fu Y, Rakugi H, Ogihara T, Tuck ML. Beta2- and beta3-adrenergic receptor polymorphisms are related to the onset of weight gain and blood pressure elevation over 5 years. Circulation. 2005;111(25):3429-3434. doi:10.1161/ CIRCULATIONAHA.104.519652.

11. Okay DM, Jackson P V., Marcinkiewicz M, Papino MN. Exercise and Obesity. Prim Care - Clin Off Pract. 2009;36(2):379-393. doi:10.1016/j.pop.2009.01.008.

12. Wanjek M, Senner V, Scharhag-Rosenberger F, Halle M. Effects of different weight loss intervention programmes in health clubs - an observational multicenter study. Eur J Sport Sci. 2016;16(7):859867. doi:10.1080/17461391.2016.1139628.

13. Strong WB, Malina RM, Blimkie CJR, Daniels SR, Dishman RK, Gutin B, Hergenroeder AC, Must A, Nixon PA, Pivarnik JM, Rowland T, Trost S, Trudeau F. Evidence based physical activity for school-age youth. J Pediatr. 2005;146(6):732-737. doi:10.1016/j.jpeds.2005.01.055.

14. Lopes WA, Leite N, da Silva LR, Brunelli DT, Gáspari AF, Radominski RB, Chacon-Mikahil, Cavaglieri CR. Effects of 12 weeks of combined training without caloric restriction on inflammatory markers in overweight girls. J Sports Sci. 2016;34(20):1902-1912. doi:10.1080/02640414.2016.1142107.

15. Leite N, Milano GE, Cieslak F, Lopes W a., Rodacki a., Radominski RB. Effects of physical exercise and nutritional guidance on metabolic syndrome in obese adolescents. Rev Bras Fisioter. 2009;13(1):73-81. doi:10.1590/S1413-35552009005016.

16. Lee S, Kim Y. Effects of exercise alone on insulin sensitivity and glucose tolerance in obese youth. Diabetes Metab J. 2013;37(4):225-232. doi:10.4093/dmj.2013.37.4.225.

17. Thompson PD, Tsongalis GJ, Seip RL, Bilbie C, Miles M, Zoeller R, Visich P, Gordon P, Angelopoulos TJ, Pescatello L, Bausserman L, Moyna N. Apolipoprotein E Genotype and Changes in Serum Lipids and Maximal Oxygen Uptake with Exercise 
Training. Metabolism. 2004;53(2):193-202. doi:10.1016/j. metabol.2003.09.010.

18. Marti A, Corbalán MS, Martínez-Gonzalez MA, Martinez JA. TRP64ARG polymorphism of the beta 3-adrenergic receptor gene and obesity risk: effect modification by a sedentary lifestyle. Diabetes Obes Metab. 2002;4(6):428-430. http://www.ncbi.nlm. nih.gov/pubmed/12406043. Accessed June 21, 2016.

19. Meirhaeghe a, Helbecque N, Cottel D, Amouyel P. Impact of polymorphisms of the human beta2-adrenoceptor gene on obesity in a French population. Int J Obes Relat Metab Disord. 2000;24(3):382-387. http://www.ncbi.nlm.nih.gov/ pubmed/10757635.

20. de Mello MT, Piano A, Carnier J, Sanches PL, Corrêa FA, Tock L, Ernandes RMY, Tufik S, Dâmaso AR. Long-term effects of aerobic plus resistance training on the metabolic syndrome and adiponectinemia in obese adolescents. J Clin Hypertens (Greenwich). 2011;13(5):343-350. doi:10.1111/j.1751-7176.2010.00388.x.

21. Dâmaso AR, Campos RMDS, Caranti DA, Piano A, Fisberg M, Foschini D, Sanches PL, Tock L, Lederman HM, Tufik S, De Mello MT. Aerobic plus resistance training was more effective in improving the visceral adiposity, metabolic profile and inflammatory markers than aerobic training in obese adolescents. J Sports Sci. 2014;32(June):1-11. doi:10.1080/02640414.2014.900692.

22. Libardi CA, De Souza GV, Cavaglieri CR, Madruga VA, ChaconMikahil MPT. Effect of resistance, endurance, and concurrent training on TNF- $\alpha$, IL-6, and CRP. Med Sci Sports Exerc. 2012;44(1):50-56. doi:10.1249/MSS.0b013e318229d2e9.

23. Lohman TG, Roche AF, Martorell R. Anthropometric Standardization Reference Manual. Vol 24. 1988. http://books. google.com/books?id=jjGAAAAAMAAJ\&pgis=1.

24. Coutinho PR, Leite N, Lopes WA, Da Silva LR, Consentino CM, Araújo CT, Moraes Jr FB, Jesus IC, Cavaglieri CR, Radominski RB. Association between adiposity indicators, metabolic parameters and inflammatory markers in a sample of female adolescents. Arq Bras Endocrinol Metabol. 2015;59(4):325-334. doi:10.1590/2359-3997000000070.

25. Brown LE, Weir JP. Accurate Assessment of Muscular Strength and Power JEPonline Journal of Exercise Physiologyonline ASEP Procedures Recommendation ASEP PROCEDURES RECOMMENDATION I: ACCURATE ASSESSMENT OF MUSCULAR STRENGTH AND POWER. An Int Electron J. 2001;4(3):1-21.

26. Jones AM, Doust JH. A $1 \%$ treadmill grade most accurately reflects the energetic cost of outdoor running. J Sports Sci. 1996;14(4):321-327. doi:10.1080/026404196367796.

27. Friedewald WT, Levy RI, Fredrickson DS. Estimation of the concentration of low-density lipoprotein cholesterol in plasma, without use of the preparative ultracentrifuge. Clin Chem. 1972;18(6):499-502. http://www.ncbi.nlm.nih.gov/ pubmed/4337382. Accessed April 12, 2016.

28. Matthews DR, Hosker JP, Rudenski AS, Naylor BA, Treacher DF, Turner RC. Homeostasis model assessment: insulin resistance and beta-cell function from fasting plasma glucose and insulin concentrations in man. Diabetologia. 1985;28(7):412-419. http://www. ncbi.nlm.nih.gov/pubmed/3899825. Accessed April 12, 2016.

29. Katz A, Nambi SS, Mather K, Baron AD, Follmann DA, Sullivan G, Quon MJ. Quantitative insulin sensitivity check index: a simple, accurate method for assessing insulin sensitivity in humans. J Clin Endocrinol Metab. 2000;85(7):2402-2410. doi:10.1210/jcem.85.7.6661.

30. Lahiri DK, Nurnberger JI. A rapid non-enzymatic method for the preparation of HMW DNA from blood for RFLP studies. Nucleic Acids Res. 1991;19(19):5444. http://www.ncbi.nlm.nih. gov/pubmed/1681511. Accessed June 8, 2016.

31. Vlachos IS, Hatziioannou A, Perelas A, Perrea DN. Sonographic assessment of regional adiposity. AJR Am J Roentgenol. 2007;189(6):1545-1553. doi:10.2214/AJR.07.2366.

32. Majem LLS, Barba L. Recordatorio de 24 horas. In: Majem LLS, Bartrina JA, Verdú JM, eds. Nutrición Y Salud Pública - Métodos, Bases Científicas Y Aplicaciones. Vol Barcelona: masson; 1995.

33. Cohen J. Statistical Power Analysis. Curr Dir Psychol Sci. 1992;1(3):98-101. doi:10.1111/1467-8721.ep10768783.

34. Mattevi VS, Zembrzuski VM, Hutz MH. Impact of variation in ADRB2, ADRB3, and GNB3 genes on body mass index and waist circumference in a Brazilian population. Am J Hum Biol. 2006;18(2):182-186. doi:10.1002/ajhb.20486.

35. Ochoa MC, Moreno-Aliaga MJ, Martínez-González MA, Martínez JA, Marti A. TV watching modifies obesity risk linked to the $27 \mathrm{Glu}$ polymorphism of the ADRB2 gene in girls. Int J Pediatr Obes. 2006;1(2):83-88. doi:http://dx.doi. org/10.1080/17477160600650386.

36. Leite N, Lazarotto L, Milano GE, Titski ACK, Consentino CLM, Mattos F, Andrade FA, Furtado-Alle L. Associação do gene ADRB2 com sobrepeso e asma em crianças e adolescentes e sua relação com a aptidão física. Rev Paul Pediatr. 2015;33(4):381386. doi:10.1016/j.rpped.2015.01.012.

37. Consentino CLM, Furtado-Alle L, Silva LR Da, Lopes WA, Tureck LV, Milano GE, Lazarotto L, Cavaglieri CR, Leite N. Influência dos polimorfismos no receptor beta 2 adrenérgico na presença de broncoespasmo induzido pelo exercício em adolescentes. Rev Paul Pediatr. 2015;(xx). doi:10.1016/j. rpped.2015.06.002.

38. Rauhio A, Uusi-Rasi K, Nikkari ST, Kannus P, Sievänen H, Kunnas T. Association of the FTO and ADRB2 genes with body composition and fat distribution in obese women. Maturitas. 2013;76(2):165-171. doi:10.1016/j.maturitas.2013.07.004.

39. Podolsky RH, Barbeau P, Kang H-S, Zhu H, Treiber FA, Snieder H. Candidate genes and growth curves for adiposity in African- and European-American youth. Int J Obes (Lond). 2007;31(10):1491-1499. doi:10.1038/sj.ijo.0803673.

40. Leite N, Milano-Gai, GE., Lazarotto L, Milano GE, Radominski, RB. Andrade F, Furtado-Alle L. Overweight adolescents and $\beta 2-$ adrenergic receptor gene polymorphisms: metabolic response after 12-weeks aerobic training. In: 19th Annual Congress of the EUROPEAN COLLEGE OF SPORT SCIENCE. Vol ; 2014:679.

41. Iyer SS, Cheng G. Role of interleukin 10 transcriptional regulation in inflammation and autoimmune disease. Crit Rev Immunol. 2012;32(1):23-63. http://www.ncbi.nlm.nih.gov/ pubmed/22428854. Accessed June 28, 2016.

42. Bea JW, Lohman TG, Cussler EC, Going SB, Thompson PA. Lifestyle modifies the relationship between body composition and adrenergic receptor genetic polymorphisms, ADRB2, ADRB3 and ADRA2B: a secondary analysis of a randomized controlled 
trial of physical activity among postmenopausal women. Behav Genet. 2010;40(5):649-659. doi:10.1007/s10519-010-9361-1.

43. Scheller J, Chalaris A, Schmidt-Arras D, Rose-John S. The proand anti-inflammatory properties of the cytokine interleukin-6. Biochim Biophys Acta - Mol Cell Res. 2011;1813(5):878-888. doi:10.1016/j.bbamcr.2011.01.034.

44. Gleeson M, Bishop NC, Stensel DJ, Lindley MR, Mastana SS, Nimmo MA. The anti-inflammatory effects of exercise: mechanisms and implications for the prevention and treatment of disease. Nat Rev Immunol. 2011;11(9):607-615. doi:10.1038/nri3041; 10.1038/nri3041.

45. Cao H. Adipocytokines in obesity and metabolic disease. J Endocrinol. 2014;220(2):T47-59. doi:10.1530/JOE-13-0339.

46. Daghestani MH, Warsy A, Daghestani MH, Al-odaib AN, Eldali A, Al-Eisa NA, Al-zhrani S. The Gln27Glu polymorphism in $\beta 2$-adrenergic receptor gene is linked to hypertriglyceridemia, hyperinsulinemia and hyperleptinemia in Saudis. Lipids Health Dis. 2010;9:90. doi:10.1186/1476-511X-9-90.

47. Cipolletta E, Carillo A, Annunziata R, Trimarco B, Franco A, Iaccarino $\mathrm{G}$. The impact of (beta) 2 adrenergic receptor polymorphisms on the outcomes in cardiovascular diseases. Cardiogenetics. 2014;4(1):10-21. doi:10.4081/cardiogenetics.2014.4661.

48. Atala MM, Consolim-colombo FM. Influência dos polimorfismos dos genes dos receptores $\beta$-adrenérgicos na regulação cardiovascular e no desenvolvimento das doenças cardiovasculares. Receptor. 2007;14(4):258-264.

49. Malina RM, Bouchard C, Bar-Or O. Growth, Maturation, and Physical Activity.; 2004.

\section{Acknowledgements}

“This work was partial supported by Fundação Araucária do Estado do Paraná under Grant 19281-408/2012”. Leite N and Cavaglieri CR are productivity scholarship holders from CNPq; Silva LR is a scholarship holder from CAPES, FurtadoAlle L is a productivity scholarship holder from Fundação Araucária.

\section{Corresponding author}

Neiva Leite

Department of Physical Education - Federal University of Paraná, Curitiba-PR, Bra-

zil. Adress: Rua Coração de Maria, 92. Jardim Botânico, Curitiba, Paraná, Brazil.

Email: neivaleite@gmail.com

Manuscript received on June 29, 2017

Manuscript accepted on September 14, 2017

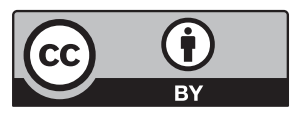

Motriz. The Journal of Physical Education. UNESP. Rio Claro, SP, Brazil

- eISSN: 1980-6574 - under a license Creative Commons - Version 3.0 